\section{INJURY PREVENTION STRATEGIES IN PORTUGUESE YOUTH SOCCER: PERCEPTIONS OF U14 MALE PRACTITIONERS}

1,2Mário A Rodrigues-Ferreira, ${ }^{1,2}$ António VencesBrito, ${ }^{1,2}$ Félix Romero, ${ }^{1,2}$ Nuno Loureiro,
${ }^{3,4}$ Maria António Castro. ' 'Sport Sciences School of Rio Maior, Polytechnic Institute of
Santarém, Rio Maior, Portugal; ' ${ }^{2}$ Life Quality Research Center - CIEQV, Santarém, Portugal;
${ }^{3}$ School of Health Sciences, Polytechnic of Leiria, Leiria, Portugal; ' ${ }^{4}$ University of Coimbra,
CEMMPRE, Dep. Eng. Mec., Coimbra, Coimbra, Portugal

10.1136/bjsports-2021-IOC.193

Background Athletes' knowledge about sports injuries can influence the adoption of prevention strategies aimed at reducing the incidence of injuries.

Objective The aim of the study was to investigate the perceptions of U14 male soccer players about the strategies for the prevention of injuries.

Design Sports injury prevention strategies survey.

Setting Youth Portuguese male soccer players competing at the national or regional level.

Participants 263 U14 male soccer players volunteered and, written informed consent was obtained from the athletes and parents or guardians.

Interventions (or Assessment of Risk Factors) A questionnaire on sports injuries prevention strategies was completed by the U14 male players selected among the 22 Portuguese regional teams competing in the Inter-Associations Tournament.

Main Outcome Measurements Knowledge and beliefs of the players about the strategies for preventing soccer injuries which might be an important approach as a primary prevention measure.

Results The strategies believed to prevent injuries main reported were performing specific preparation exercises before training and games $(83,3 \%)$, pre-season physical conditioning $(73,4 \%)$, complementary soccer training physical conditioning (70\%), and nutritional counselling (49,8\%). The less reported strategies were being informed by physiotherapists about injury risks $(49,4 \%)$, having more health professionals working with the team $(41,8 \%)$; using protective equipment $(33,5 \%)$, materials of better quality $(25,1 \%)$, and material innovation $(16,3 \%)$.

Conclusions A sample of U14 Portuguese male soccer players perceived the need to perform specific preparation exercises before training and games, as well as physical conditioning that complements soccer training, in the pre-season and during the season, as the main measures to prevent injuries in youth soccer. Evidence shows that the strategies identified by the athletes have a beneficial impact on injury prevention. Future studies should analyse the relationship between the incidence and mechanism of injury and preventive strategies in Portuguese youth soccer.

\section{GENDER-SPECIFIC DIFFERENCES IN NEUROMUSCULAR ACTIVATION IN THE KNEE STABILIZING MUSCLES IN ADULTS - A SYSTEMATIC REVIEW}

${ }^{1}$ Martina Steiner, ${ }^{1}$ Heiner Baur, ${ }^{1,2}$ Angela Blasimann. ${ }^{1}$ Bern University of Applied Sciences, Department of Health Professions, Discipline of Physiotherapy, Bern, Switzerland; ${ }^{2}$ University of Antwerp, Faculty of Medicine and Health Sciences, Department of Rehabilitation Sciences and Physiotherapy, Antwerp, Belgium

\subsection{6/bjsports-2021-IOC.194}

Background Women have a higher injury rate for anterior cruciate ligament ruptures than men. Various indicators for this gender-specific difference are controversially discussed.
Objective To find out if there is a gender-specific difference in neuromuscular activation of the knee stabilizing muscles in adult female and male subjects measured with surface electromyography (EMG).

Design Systematic literature review, registered in PROSPERO (CRD42020189504).

Setting PubMed, CINAHL, Embase, Cochrane and SPORTDiscus were searched from inception until September 2020 including e-mail alerts. The quality of included studies was assessed according to the National Heart, Lung and Blood Institute study quality assessment tool.

Patients (or Participants) Healthy female and male subjects $(\geq$ 18 years of age).

Interventions (or Assessment of Risk Factors) Neuromuscular activation of the knee stabilizing muscles in different task (e.g. cutting manoeuvres) comparing female and male subjects.

Main Outcome Measurements Outcome measures describing neuromuscular activation of the knee stabilizing muscles with amplitude magnitude and time domain variables.

Results A total of 2'612 articles were identified. After deduplication, 1'802 articles were screened for title and abstract. Sixty-five articles were fully read and assessed for eligibility. Finally, a total of 15 articles, all cross-sectionally designed, were included in the qualitative synthesis. The methodological quality of the studies was mostly rated 'fair' (40\%). A significantly higher activity of the quadriceps muscle in females was found in three studies. Two studies found significantly lower neuromuscular activity in the hamstrings in females. The remaining studies found no significant difference or even contradicting results.

Conclusions The controversial findings do not allow for a concluding answer to the question of a gender-specific neuromuscular activation. Further investigations with higher statistical power and a more homogeneous methodological approach (tasks and data normalization) of the included studies may help to gain better insight into any gender-specific differences in neuromuscular activation that may exist.

\section{IS IT POSSIBLE TO PREVENT RUNNING RELATED INJURIES IN ADULT NOVICE RUNNERS WITH AN ONLINE BEHAVIORAL INTERVENTION?}

${ }^{1}$ Henrike van der Does, ${ }^{2,3,4}$ Vincent Gouttebarge, ${ }^{1}$ Ellen Kemler. ${ }^{1}$ Dutch Consumer Safety Institute, Amsterdam, Netherlands; ${ }^{2}$ Amsterdam UMC, University of Amsterdam, Department of Orthopaedic Surgery, Amsterdam Movement Sciences, Amsterdam, Netherlands; ${ }^{3}$ Section Sports Medicine, University of Pretoria, Pretoria, South Africa; ${ }^{4}$ Amsterdam Collaboration on Health and Safety in Sports (ACHSS), Amsterdam $10 \mathrm{C}$ Research Center of Excellence, Amsterdam, Netherlands

\subsection{6/bjsports-2021-IOC.195}

Background Novice runners have a high injury risk. The online behavioral intervention Runfitcheck was effective in stimulating preventive behavior in novice runners, but its effectiveness on running-related injuries (RRI) remains unknown.

Objective To evaluate the effectiveness of the Runfitcheck on RRIs among adult novice runners.

Design A three-arm randomized controlled trial was conducted over a period of seven months.

Setting Competitive level: novice runners.

Participants A total of 851 participants of 18 years or older, considering themselves as inexperienced, slightly or rather experienced runners or having less than one year running 